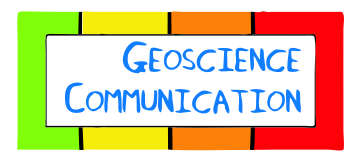

Discussions

Interactive comment on "“"Thanks for helping me find my enthusiasm for physics!' The lasting impacts "research in schools" projects can have on students, teachers, and schools" by Martin O. Archer and Jennifer DeWitt

Martin O. Archer and Jennifer DeWitt

m.archer10@imperial.ac.uk

Received and published: 22 November 2020

This paper is very interesting and it presents, in a very well organised and clear manner, the impact of a "Research in Schools" programme on participating students, teachers and their schools. The study was well designed and the authors draw very honest conclusions from the acquired data and from its analysis, in particular keeping the conclusions specific to the cohorts that participated in the programme. While nontrivial, the inclusion of control groups in the design would have added an extra depth to the study. Perhaps that will be possible in 


\section{future studies.}

We thank the reviewer for their time in assessing the manuscript. In our companion paper, M.O. Archer et al. (2020), we discussed the ethical reasoning behind a lack of control groups in our evaluation of the programme. We will mention this limitation to the study and add a reference to the reasoning in the manuscript as follows:

No control groups were established due to ethical considerations, further explored in M.O. Archer et al. (2020), which slightly limits this impact study. However, where possible we draw from publicly available benchmark data.

Archer, M. O., DeWitt, J., and Thorley, C.: Transforming school students' aspirations into destinations through extended interaction with cutting-edge research: Physics Research in School Environments, Geosci. Commun. Discuss., https://doi.org/10.5194/gc-2020-35, in review, 2020.

Regarding the results presented on section 4.1.2 Skills, I am left wondering if students may have been influenced by teachers and/or by introductory materials handed out to them at the start of their participation in PRiSE, in building the list of developed skills. Was a list of "skills to be developed. . ." provided to them in order to "sell" the project? I suggest to include a comment on that.

The reviewer raises an interesting point. None of the resources provided with the projects indicated expected skills to be developed, however, we cannot comment on what teachers may have said to their students outside of interventions. We will make the following addition:

Skills development was not mentioned in any of the PRiSE projects' resources (e.g. there was no list of "skills to be developed") so we are confident in the validity of these results, though we cannot rule out that teachers may have influenced students' answers. 
On section 6 Conclusions, on line 538, the authors state ". . .suggest that these projects have been highly memorable. . .”. I agree that the data fully supports that the projects were memorable, but the classification as highly memorable seem a more subjective opinion by the authors. I suggest leaving out "highly" on that sentence.

We will make this change.

The citation included in the title, while making it catchier, introduces some unnecessary bias. A different citation could have been, e.g., "I never really saw Physics as a choice for me . . . and the project hasn't changed my mind about this." Perhaps it is better to leave any citation out of the title. Having said that, I thoroughly recognise that the authors' choice of citation is a much better reflection of the observed overall trend than the example I use above.

The reviewer is correct that the quote used in the title was designed to make it catchier. We believe the quote exemplifies the overall quantitative and qualitative results of the paper, as the reviewer points out, that PRiSE students' physics aspirations (after 6 months) and university destinations (after 3 years) were enhanced and that physics teachers' practice was developed. Therefore, we feel its usage is appropriate in summarising the paper's conclusions.

I cannot finish without stressing the importance of this and of similar studies. They are crucial to better understand the real impact of science communication activities carried out by HE institutions, research centres, science centres and museums, and to guide institutions and individuals into better practices.

We thank the reviewer for this comment.

P.S. The sentence beginning towards the end of line 244 reads "A similar proportion to with physics of $68 \%$...". Being a non-native English speaker, I may be missing some nuance of the English language, but that sentence doesn't sound

Printer-friendly version

Discussion paper 
right to me. Apologies if it is my fault.

We will adjust this sentence to make it clearer as follows

GCD

$68 \pm 9 \%$ of students indicated PRiSE's likely positive influence on their STEM aspirations (a result similar to physics aspirations).

Interactive

comment

Interactive comment on Geosci. Commun. Discuss., https://doi.org/10.5194/gc-2020-36, 2020. 